\title{
CIBERINFÂNCIA: UMA ANÁLISE SOBRE O BINÔMIO CRIANÇA E JOGOS ELETRÔNICOS
}

Leonardo de Angelo Orlandi, José Milton de Lima, Márcia Regina Canhoto de Lima

Universidade Estadual Paulista - UNESP, Programa de Pós-Graduação em Educação, Presidente Prudente, SP.Email:leo angeloorlandi@hotmail.com.

\section{RESUMO}

A presente pesquisa tem como foco as sensações e percepções causadas em crianças do ensino fundamental pelo uso de jogos e dispositivos eletrônicos. Para tanto, estabeleceu-se como objetivo: realizar uma revisão sistemática da literatura a respeito de estudos que procurem caracterizar o uso de jogos eletrônicos e as sensações produzidas em crianças do ensino fundamental, que auxiliem na compreensão e na elaboração de novos critérios. A metodologia utilizada foi a revisão sistemática de literatura e análise de conteúdo. A fim de lançar luz sobre um objeto que se destaca com tamanha relevância no campo educacional, reiteramos a necessidade de compreender as sensações produzidas pelos jogos e dispositivos eletrônicos e como se apresentam no ambiente escolar.

Palavras-chave: Jogos eletrônicos; Ensino Fundamental; Infância.

\section{CIBERCHILDWOOD: AN ANALYSIS OF THE BINOMIO CHILD AND ELECTRONIC GAMES}

\begin{abstract}
This research is focused on the sensations and perceptions caused in elementary school children through the use of games and electronic devices. Therefore, it established itself as objective: to conduct a systematic review of the literature on studies that seek to characterize the use of electronic games and the sensations produced in elementary school children, to assist in the understanding and development of new criteria. The methodology used was the systematic review of literature and content analysis. In order to shed light on an object that stands out with such relevance in the educational field, we reiterate the need to understand the sensations produced by the games and electronic devices and they are presented in the school environment.
\end{abstract}

Keywords: Electronic games; Elementary School; Childhood. 


\section{INTRODUÇÃO}

$\mathrm{Na}$ contemporaneidade, é comum os pesquisadores se preocuparem com a análise da produção científica, uma vez que essa apreensão é importante em todas as áreas de conhecimento. Tal apreciação permite a identificação e uma sistematização dos temas já estudados, fornecendo as implicações destes estudos para as novas necessidades científicas. Além disso, é possível analisar também os pontos de vista metodológicos, a fim de que novos aspectos teóricos sejam construídos e auxiliem na compreensão da realidade. É nessa direção que a revisão desta tese pretende contribuir.

Para tanto, optamos por uma análise da produção cientifica na área de educação e tecnologia, com foco para o uso de jogos eletrônicos por crianças do ensino fundamental. No entanto, delimitamos nosso estudo a fim de que o mesmo abordasse, no contexto da cibercultura, sensações produzidas pelos jogos eletrônicos em crianças em idade escolar. $O$ estudo sobre os efeitos dos jogos eletrônicos é um tema bastante presente na literatura científica. Isso indica a sua relevância e, possivelmente, a sua inesgotável discussão no que se refere às análises necessárias destes aspectos. Às vezes, corre-se o risco de soar como tema repetitivo, no entanto, essa presença tão forte na literatura pode indicar também uma necessidade de aprofundamento sobre as mudanças na sociedade no que diz respeito às tecnologias.

Para Abreu et al (2008), com o grande avanço tecnológico das últimas décadas, principalmente no que tange à eletrônica e à informática, os dispositivos móveis e os jogos eletrônicos tornaram-se cada vez mais populares. O videogame, sem dúvida alguma, passou a ser uma das mais importantes atividades de lazer para crianças e adolescentes. Diversos estudos relacionam o uso de jogos eletrônicos com a maior facilidade de aprendizado, o desenvolvimento de habilidades cognitivas e motoras, a melhora na capacidade de orientação espacial e a facilitação da socialização. As vantagens de seu uso têm sido testadas e comprovadas também em terapias médicas, incluindo psicoterapias (MENDES, 2006; MOITA, 2007).

Tais pesquisas, no entanto, são bastante heterogêneas e a variabilidade da nomenclatura e a inexistência de um consenso sobre os efeitos positivos ou negativos dos jogos eletrônicos, devido à falta de critérios diagnósticos e definições operacionais estabelecidos, são alguns dos fatores responsáveis por essa diversidade de estudos. Nesse contexto diverso, esta etapa da pesquisa busca realizar uma revisão sistemática da literatura a respeito de estudos que procurem caracterizar o uso de jogos eletrônicos e as sensações produzidas em crianças do ensino fundamental, que auxiliem na compreensão e na elaboração de novos critérios.

\section{METODOLOGIA}

Trata-se de uma revisão bibliográfica sistematizada, que abordou a temática da cibercultura, com foco para o uso de dispositivos e jogos eletrônicos por crianças da educação básica no contexto do ensino fundamental, realizada em três etapas: busca por descritores no Portal de periódicos da Coordenação de Aperfeiçoamento de Pessoal do Nível Superior (CAPES), seleção por relevância da temática e análise de conteúdo.

Para o delineamento desta revisão, na primeira etapa, realizou-se uma busca sistematizada com critérios específicos, no Portal da CAPES (Periódicos). Na segunda etapa, foi realizada uma seleção dos resumos deste estudo que contemplavam os principais critérios estabelecidos. E, por fim, foi estudado cada artigo selecionado. O levantamento realizado teve como foco o período compreendido entre 1995 e 2015. Este período foi delimitado, levando em consideração que nos últimos vinte anos os avanços nas tecnologias e na sociedade podem ter ofertado novas possibilidades de estudos. Foram reunidos artigos e teses, mas em grande parte foram 
pesquisados artigos de programas de Pós-graduação credenciados e artigos publicados com seletiva política editorial, tendo como fonte única o portal de periódicos da Capes (www. periodicos.capes.gov.br).

Para padronização e organização da busca foram utilizados os seguintes descritores: Jogos eletrônicos; Cibercultura e Educação; Jogos e Dispositivos Móveis. Durante o procedimento, foi realizada uma análise dos resumos de todos os trabalhos encontrados, excluindo-se aqueles que não sugeriram vinculação entre as expressões de jogos eletrônicos, cibercultura, crianças e educação. Para delinearmos o processo de maneira clara e concisa houve o recorte dos descritores e relevância ao tema, voltado para a realidade brasileira. Utilizamos a abordagem quantitativa, tendo como critério inicial selecionar o numero expressivo de publicações do tema em foco. Com o intuito de qualificar a apreciação, remetemo-nos a metodologia qualitativa, uma vez que após a seleção quantitativa, houve a preocupação em qualificar e selecionar os textos para posterior análise e tratamento dos dados coletados.

Nesse contexto, os critérios utilizados para seleção dos textos a serem analisados foram: a literatura estar vinculada ao uso de dispositivos e jogos eletrônicos por crianças do ensino fundamental. A análise de conteúdo norteou a explanação e reflexão sobre os dados coletados nos artigos e teses, com base nas autoras Bardin (2009). A análise de conteúdo é definida como uma técnica de tratamento de dados de pesquisa, voltada para uma análise objetiva, sistemática e quantitativa do conteúdo de "comunicações" (em nosso caso, textos). Dessa forma, ainda que tivesse um viés quantitativo, essa análise buscou aspectos qualitativos desse material selecionado.

Na visão de Bardin (2009, p. 74), a análise de conteúdo é "compreendida muito mais como um conjunto de técnicas". Na visão da autora, constitui-se na análise de informações sobre o comportamento humano, possibilitando uma aplicação bastante variada, e tem duas funções: verificação de hipóteses e/ou questões e descoberta do que está por trás dos conteúdos manifestos. Tais funções podem ser complementares, com aplicação tanto em pesquisas qualitativas como quantitativas, como neste caso que a mesma ocupa-se das duas abordagens. Após a leitura e análise minuciosas dos artigos, sistematizamos três categorias que seguiram os termos ou expressões dos descritores de busca. Foi realizada uma descrição e posterior discussão e, em seguida, buscamos as implicações dos estudos para a construção do objeto específico desta tese e, principalmente, para as novas contribuições científicas.

\section{RESULTADOS}

Inicialmente serão apresentados os dados quantitativos, estabelecendo algumas relações descritivas entre estes dados. Posteriormente, serão descritos os estudos selecionados para análise. Por fim, serão tecidas discussões com base no que foi apontado em cada pesquisa e autores complementares da área. Em uma primeira consulta, foi realizada a pesquisa tendo como descritores: Jogos eletrônicos. Sobre esse descritor foram encontrados 283 artigos, dos quais foram salvos 45 , os quais se apresentavam na temática estabelecida. Já com o Cibercultura e Educação foram encontrados 130 artigos, dos quais numa primeira seleção foram salvos 16 artigos usando o mesmo critério. Com o descritor Jogos e Dispositivos móveis foram encontrados 44 artigos e selecionados 8 para futura análise. Passemos, então, aos resultados da segunda etapa de seleção, sendo que de um total de 457 artigos, foram selecionados 69 e, por fim, 28 artigos foram analisados de modo minucioso.

Tabela 01. Distribuição dos artigos pesquisados a partir de cada descritor considerado.

\begin{tabular}{lcccc}
\hline \multicolumn{1}{c}{ Descritores } & $\begin{array}{c}\text { Total } \\
\text { de artigos }\end{array}$ & $\begin{array}{c}\text { Artigos } \\
\text { selecionados }\end{array}$ & $\begin{array}{c}\text { Artigos } \\
\text { descartados }\end{array}$ & $\begin{array}{c}\text { Artigos } \\
\text { Analisados }\end{array}$ \\
\hline Jogos Eletrônicos & 283 & 45 & 238 & 14 \\
Cibercultura e Educação & 130 & 16 & 114 & 9 \\
\hline
\end{tabular}




\begin{tabular}{ccccc}
\hline Jogos e Dispositivos móveis & 44 & 8 & 36 & 4 \\
\hline TOTAL & 457 & 69 & 388 & 27 \\
\hline É importante salientar que nessa & busca, & percebeu-se que alguns & artigos, & mesmo com
\end{tabular}
descritores diferentes, se repetiam o que é compreensível por se tratar de interfaces do mesmo tema. A distribuição percentual do número de artigos por descritor não está equilibrada, uma vez que o descritor "Jogos Eletrônicos" possui por volta de $62 \%$ dos resultados pesquisados.

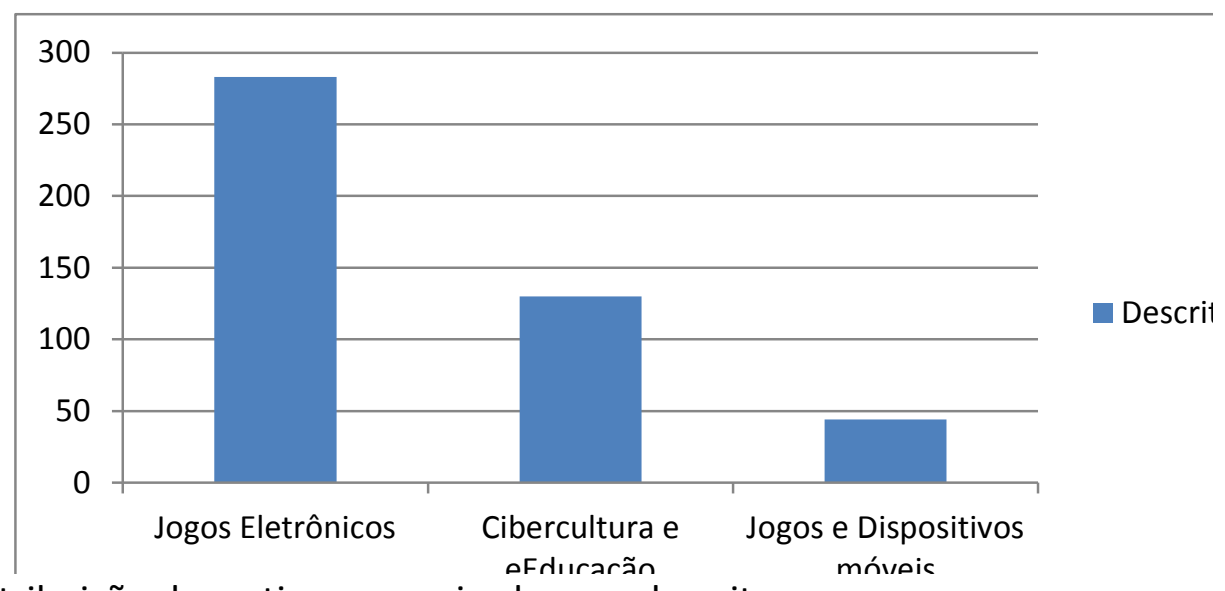

Gráfico 1. Distribuição dos artigos pesquisados por descritores.

No tocante, percebemos que a análise dos artigos seguiu a predisposição das buscas pelos descritores mantendo o descritor "Jogos Eletrônicos" com o maior número de artigos analisados nesta revisão (52\%).

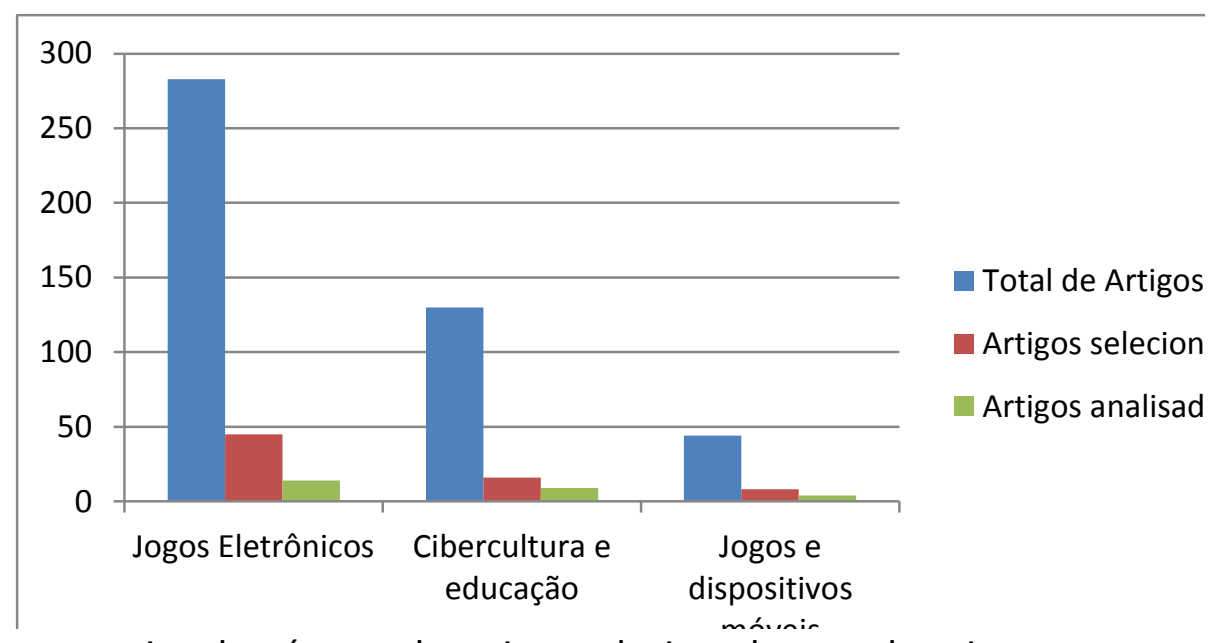

Gráfico 2. Demonstrativo do número de artigos selecionados por descritores.

No que diz respeito à distribuição temporal, 6 pesquisas (25\%) foram publicadas até o ano de 2009 e a partir de 2010 (últimos cinco anos), 18 (75\%) das pesquisas. Consideramos que o aumento expressivo das pesquisas nos últimos cinco anos está relacionado ao desenvolvimento cada vez maior de aparatos tecnológicos e de sua importância no cotidiano dos indivíduos e da sociedade. De acordo com dados da CEPAL (Comissão Econômica para a América Latina e o Caribe), a América Latina se transformou, durante a última década, em um mercado emergente no uso de programas e aplicativos tecnológicos por parte das empresas, dos governos e dos indivíduos, e o gasto nessa área alcançou 295.000 milhões de dólares em 2011, cerca de 5,2\% do PIB.

Essas transformações afetaram todas as esferas da sociedade, inclusive a área da educação e, consequentemente, as pesquisas relacionadas à temática sobre tecnologias tem ganhado destaque nos últimos cinco anos. Outro fator que influencia esse número são os debates e as 
produções de artigos que começaram a dar visibilidade à temática até então pouco explorada nos programas de Pós-graduação em educação no Brasil.

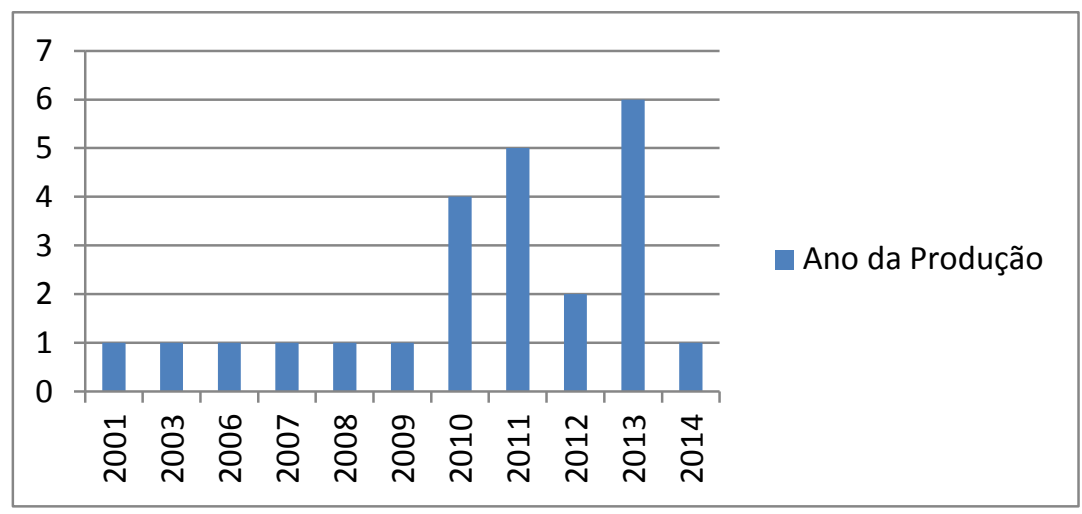

Gráfico 3. Ano da produção dos artigos sobre os jogos eletrônicos, cibercultura e educação.

No movimento de análise dos trabalhos, identificamos que a maioria das investigações utilizaram diversas técnicas de pesquisa para a coleta de dados. No entanto, ficou evidente o uso da análise bibliográfica e da observação participante na maioria das pesquisas selecionadas. Verificamos, portanto, que $73 \%$ das investigações utilizaram esses procedimentos como forma de coleta de dados. No tocante, apresentamos a análise de conteúdo dos artigos selecionados divididos por categoria. A análise procurou evidenciar os principais aspectos tratados no texto e as ideias principais relatadas pelos autores. Para tanto, os artigos apresentados a seguir foram analisados no sentido de identificar aspectos factuais que contribuiriam para o desenvolvimento da pesquisa.

\section{CONCLUSÃO}

Com o grande avanço tecnológico das últimas décadas, principalmente no que tange à eletrônica e à informática, a internet e os jogos eletrônicos tornaram-se cada vez mais populares. O videogame, sem dúvida alguma, passou a ser uma das mais importantes atividades de lazer para crianças e adolescentes. Os usuários de internet, porém, não incidem em uma faixa etária ou segmento mais específico, sendo esta utilizada amplamente por pessoas de todas as idades e todos os estratos socioeconômicos no mundo todo. Muitos estudos relacionam o uso de jogos eletrônicos com a maior facilidade de aprendizado, o desenvolvimento de habilidades cognitivas e motoras, a melhora na capacidade de orientação espacial e a facilitação da socialização.

No entanto, juntamente com o aumento na popularidade do uso da rede mundial através dos dispositivos e dos jogos eletrônicos, surgiram relatos na imprensa leiga e na literatura científica de indivíduos que estariam "dependentes" da realidade virtual da Internet e dos jogos eletrônicos. Embora esses fenômenos ainda sejam pouco estudados, a maioria dos autores sugere que o uso excessivo do videogame e da Internet pode gerar inúmeras sensações e percepções que podem levar a diferentes caminhos: de um novo transtorno psiquiátrico a formação de novas habilidades cognitivas (ABREU et al, 2008).

Nessa direção, Ferreira \& Darido (2013) indicam que um dos desafios enfrentados pela Educação é o de refletir como utilizar efetivamente as tecnologias, de modo especial os jogos eletrônicos, de tal maneira a proporcionar aos alunos uma aprendizagem significativa e contextualizada.

Para Martins \& Castro (2011), a vivência em uma sociedade tecnológica potencializa deslocamentos importantes para a infância. "Por meio da tecnologia, vemos uma infância que agora pode ter acesso a informações vindas de todas as partes do mundo, comunicar-se 
rapidamente, e vivenciar, no presente, trocas significativas, em vez de voltar-se exclusivamente para sua preparação para a idade adulta" (p. 621).

As autoras (2011) refletem sobre a articulação entre vida na escola e sua relação com a tecnologia, afirmando que existe a possibilidade de permeabilidades entre essas duas formas de subjetivação: "quando a escola se abre para esse encontro com o cotidiano tecnológico das crianças, sabendo que não pode renegar esse contexto, mas tendo o cuidado de não recorrer a uma adaptação vazia de questionamentos" (p. 632).

Ao mesmo tempo, Martins \& Castro (2011) apontam para a necessidade de manter certas tensões, para conter a rapidez do tecnológico e possibilitar uma digestão do mundo para os mais novos, tomando cuidado para não cair em um processo nostálgico. O trabalho escolar sugere uma necessidade de reflexão e de aprofundamento sobre o que é estudado; logo, a escola deveria ocupar um lugar especial na geração de conhecimentos, opondo-se à superficialidade, tanto de encontros quanto de saberes.

Diante da discussão, as autoras (2011) continuam afirmando que o espaço escolar é necessariamente plural, e incorpora referências pessoais diversas. Assim, entendem que a tecnologia acolhe e promove tais encontros, mesmo que sejam mais superficiais, mas possibilita aproximações entre os mais velhos e os mais novos, entre culturas distantes, dando um lugar de saber e de participação para quem ainda não o tinha. Indicam, ainda, que não se pode negar que existam exigências sociais em torno das tecnologias, e que, para formar cidadãos, a escola deveria sim, pensar sobre a utilização desses aparatos tecnológicos, até como forma de incluir aqueles que não têm condições financeiras de tê-los em casa.

Lidar com computadores é algo tão importante, nos dias de hoje, quanto ler, escrever e saber fazer contas. As crianças estão participando ativamente do mundo tecnológico, de uma forma ou de outra, pois este faz parte do contexto social cotidiano e atual delas. Importa saber como estão sendo subjetivadas por esta condição, que práticas sociais vão ser engendradas por este aspecto da realidade contemporânea (p. 633).

Nesse contexto, é dado um mundo às crianças, mas há o perigo de um empoderamento vazio. Esse é o papel da educação, seja escolar ou não, que está nas mãos dos mais velhos: contribuir para uma análise crítica na construção de uma historicidade entre o presente tecnológico e o passado, de modo que as tecnologias possam ser inseridas em uma perspectiva humanizadora.

\section{REFERÊNCIAS}

ABREU, Cristiano Nabuco de; KARAM, Rafael Gomes; GOES, Dora Sampaio; SPRITZER, Daniel Tornaim. Dependência de Internet e de jogos eletrônicos: uma revisão. Rev. Bras.

Psiquiatr. [online]. 2008, vol.30, n.2, pp.156-167.

BARDIN, L. Análise de conteúdo. Lisboa: Edições 70, 1977.

FERREIRA, Aline Fernanda; DARIDO, Suraya Cristina. Os Jogos digitais como apoio pedagógico nas aulas de educação física escolar. In: DARIDO, S. C.; RANGEL, I. C. A. (coord.) Educação física na escola: implicações para a prática pedagógica. Rio de Janeiro: Guanabara Koogan, 2005. p.122136 .

MARTINS, L. T.; CASTRO, L. R. de (2011). Crianças na contemporaneidade: entre as demandas da vida escolar e da sociedade tecnológica. Revista Latinoamericana de Ciencias Sociales, Niñez y Juventud, 2 (9), pp. $619-634$

MENDES, Cláudio L. Jogos eletrônicos: diversão, poder e subjetivação. Campinas: Papirus, 2006. 
MOITA, Filomena. Game on: jogos eletrônicos na escola e na vida da geração @. Campinas: SP: Editora Alínea, 2007.

MURRAY, Janet H. Hamlet no holodeck: o futuro da narrativa no ciberespaço. São Paulo: Itaú Cultural: Unesp, 2003. 